\title{
Menstrual Phase and the Vascular Response to Acute Resistance Exercise In Women
}

Augustine, J., Nunemacher, K., Heffernan, K. Syracuse University, Syracuse, NY

Aerobic exercise has a favorable effect on arterial stiffness and reduces the risk for cardiovascular disease (CVD). The effects of resistance exercise (RE) on arterial stiffness are less clear. Acute RE increases central stiffness and decreases peripheral stiffness in men. However, cyclic hormonal changes in women may modulate the vascular response to RE. PURPOSE: To examine the effect of acute RE on central and peripheral arterial stiffness in women during the early follicular and the ovulatory phase of the menstrual cycle. METHODS: Eighteen healthy women (28 77 years, Body Mass Index (BMI) $22.6 \pm 2.9 \mathrm{~kg} / \mathrm{m}^{2}$ ) completed an acute RE bout during the early follicular and the ovulatory phase of their menstrual cycle. Salivary $17 \beta$-Estradiol concentration was measured during each phase, using a passive drool technique. Pulse-Wave Velocity (PWV) was obtained from the carotid-femoral and carotid-radial pulse sites to measure central and peripheral arterial stiffness, respectively, using applanation tonometry. PWV was measured at rest, immediately, 10, 20 and 30 minutes post-RE. RESULTS: $17 \beta$-Estradiol concentration was significantly lower in the early follicular versus the ovulatory phase of the menstrual cycle $(1.78 \pm 0.51 \mathrm{pg} / \mathrm{ml}$ vs. $2.40 \pm 0.26 \mathrm{pg} / \mathrm{ml}, \mathrm{p}=0.01)$. Central PWV significantly increased $(p<0.05)$ and peripheral PWV significantly decreased $(p<0.05)$ post-RE in both early follicular and ovulatory phases. No phase-by-time interaction was detected for either vascular segment (Table, $\mathrm{p}>0.05$ ). CONCLUSION: Menstrual cycle phase may not influence the vascular response to acute RE, even though there are significant differences in $17 \beta$-Estradiol concentrations between menstrual phases.

Supported by NIH NIA P30 AG0344645 05 (KSH).

\begin{tabular}{llccccc}
\hline Variable & Condition & Baseline & Post-1 & $\mathbf{1 0} \mathbf{~ m i n}$ & $\mathbf{2 0} \mathbf{~ m i n}$ & $\mathbf{3 0} \mathbf{m i n}$ \\
\hline CF-PWV (m/s) & Early Follicular & $5.6 \pm 0.8$ & $5.8 \pm 0.7^{\mathrm{a}}$ & $5.8 \pm 0.8^{\mathrm{a}}$ & $5.7 \pm 0.7$ & $5.8 \pm 0.7^{\mathrm{a}}$ \\
& Ovulation & $5.6 \pm 0.6$ & $5.9 \pm 0.7^{\mathrm{a}}$ & $6.1 \pm 0.8^{\mathrm{a}}$ & $6.1 \pm 0.9^{\mathrm{a}}$ & $6.0 \pm 0.9^{\mathrm{a}}$ \\
CR-PWV (m/s) & Early Follicular & $7.9 \pm 0.3$ & $6.8 \pm 0.3^{\mathrm{a}}$ & $7.2 \pm 0.3^{\mathrm{a}}$ & $7.8 \pm 0.3$ & $7.7 \pm 0.4$ \\
& Ovulation & $7.9 \pm 0.3$ & $6.8 \pm 0.2^{\mathrm{a}}$ & $7.1 \pm 0.3^{\mathrm{a}}$ & $7.6 \pm 0.3$ & $7.3 \pm 0.3$ \\
MAP (mmHg) & Early Follicular & $79 \pm 7$ & $83 \pm 9^{\mathrm{a}}$ & $80 \pm 10$ & $76 \pm 10$ & $79 \pm 9$ \\
& Ovulation & $78 \pm 6$ & $79 \pm 6$ & $75 \pm 7^{\mathrm{a}}$ & $76 \pm 6$ & $76 \pm 8$ \\
\hline
\end{tabular}

${ }^{a}$ Significantly different from baseline (time effect), $\mathrm{p}<0.05$; No significant phase effect; CFPWV, Carotid-femoral Pulse Wave Velocity; CR-PWV, Carotid-radial Pulse Wave Velocity; MAP, Mean Arterial Pressure. 